\title{
Are output measurements always necessary after CT tube replacement?
}

\author{
Paul J Stauduhar, Aaron Kyle Jones \\ The University of Texas M. D. Anderson Cancer Center, Houston, TX, USA.
}

Received March 19, 2014; Published Online April 08, 2014

[Presented at the Young Investigator's Symposium at the 2014 Annual Meeting of Southwest Chapter of American Association of Physicists in Medicine (AAPM) in San Antonio, Texas, USA]

\section{Conference Proceeding}

\begin{abstract}
Purpose: TX regulations and the ACR require that CT radiation output be measured within 30 days of major service. The most common major service is tube replacement. We hypothesized that historical QC data could be used instead to determine if output measurements are necessary, reducing the need for costly output measurements.

Methods: We reviewed 66 records of tube replacements to determine with what frequency output falls outside specifications. We also conducted an experiment to verify that clinically significant output changes could be identified by comparing image noise in historical $\mathrm{QC}$ data with the same data after tube replacement. We used 30 days of historical QC data to establish a baseline noise level and 95\% confidence interval (CI) for individual noise measurements. To simulate output changes, we acquired phantom images with our QC protocol while manually changing output (mA). We acquired 10 images using the baseline output and 10 images at each different "output". We evaluated individual images and subsets of images at each "output" to determine if the system was within the manufacturer's specifications.
\end{abstract}

Results: None of the 66 tube replacements resulted in an output change that exceeded specifications. Analysis of 30 days of historic QC data for our experimental system indicated a mean noise of $5.4 \mathrm{HU}$ with $95 \%$ CI of $5.1-5.7 \mathrm{HU}$. When using the mean noise of 10 images acquired at each of the varying outputs, we were able to identify, with $100 \%$

Presenting author: Paul J Stauduhar; The University of Texas M. D. Anderson Cancer Center, Houston, TX, USA.

Cite this article as:

P Stauduhar, A Jones. Are output measurements always necessary

after CT tube replacement? Int J Cancer Ther Oncol 2014;

2(2):020238. DOI: $10.14319 /$ ijcto.0202.38 accuracy, images acquired at outputs outside manufacturer's specifications.

Conclusion: The results of our review of historical tube replacement data indicated the likelihood of output falling outside manufacturer's specifications is low. Considering this, it is likely that by using QC data from programs required by regulation and the ACR physicists can reliably verify radiation output stability remotely instead of making physical measurements. 


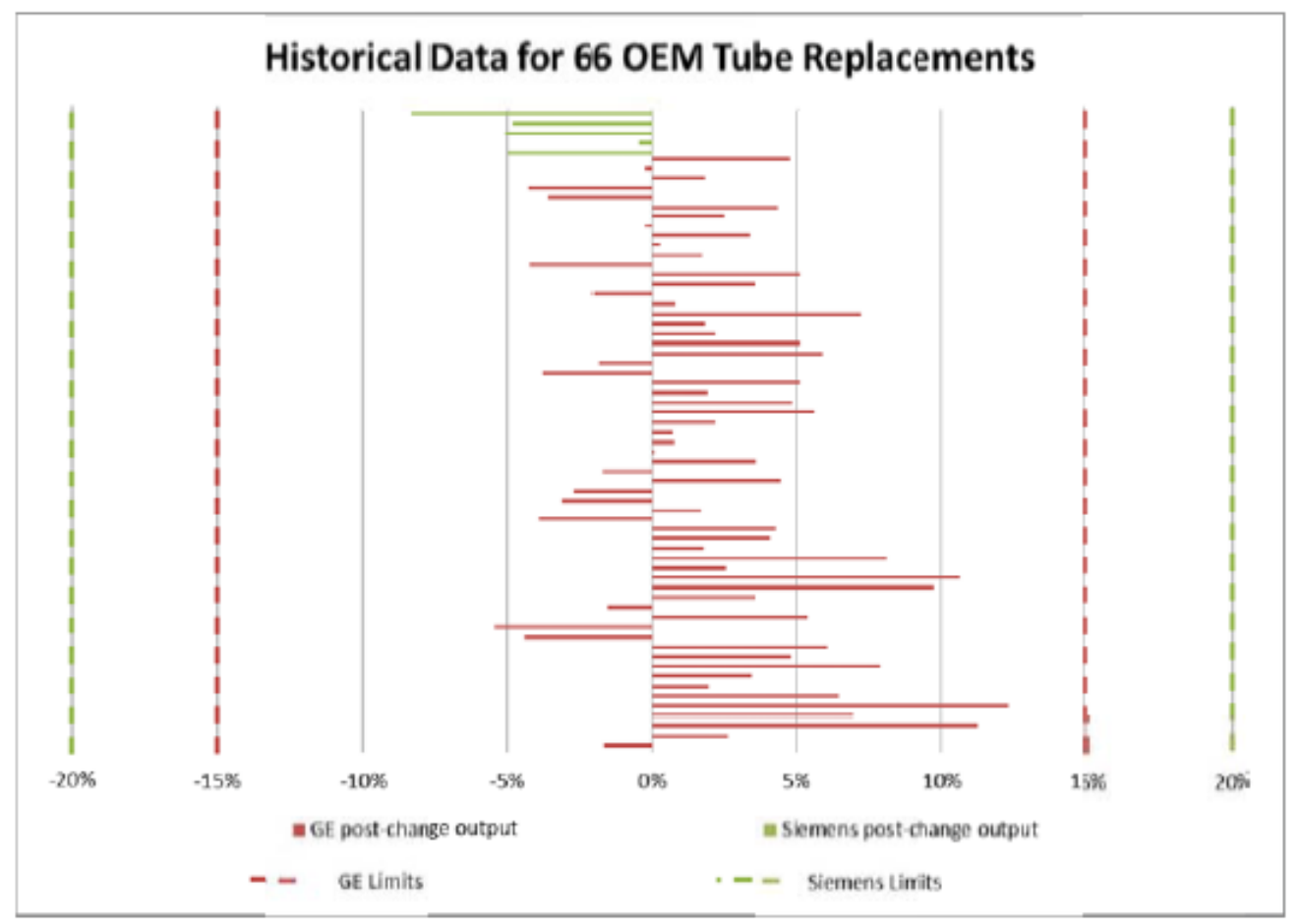

FIG.1: Plot showing output after a CT Xray tube change. Output specifications (0\%) and limits (red and green dashed lines) are from manufacturer technical specifications. The limit for Siemens was $+/ 20 \%$, GE publishes an expected variation of $+/ .15 \%$ and a maximum variation of $+/-40 \%$. In the current study (66 instances), we found no records of X ray tubes failing to meet manufacturer's specifications when OEM replacement tubes are used.

\begin{tabular}{|l|c|c|c|c|c|c|c|c|c|c|c|c|c|c|c|c|c|c|c|c|c|c|c|c|c|}
\hline $\begin{array}{l}\text { Output } \\
\text { change (\%) }\end{array}$ & -50 & -46 & -41 & -37 & -33 & -28 & -24 & -20 & -15 & -11 & -7 & -2 & 0 & 2 & 7 & 11 & 15 & 20 & 24 & 28 & 33 & 37 & 41 & 46 & 50 \\
\hline $\begin{array}{l}\text { Image noise } \\
\text { Within +/- }\end{array}$ & 7.7 & 7.4 & 7.1 & 6.7 & 6.4 & 6.3 & 6.2 & 6.1 & 5.9 & 5.6 & 5.6 & 5.5 & 5.3 & 5.4 & 5.3 & 5.1 & 5.1 & 5.0 & 4.8 & 4.8 & 4.7 & 4.6 & 4.5 & 4.5 & 4.3 \\
\hline $\begin{array}{l}15 \% \\
\text { output?+ }\end{array}$ & Fail & Fail & Fail & Fail & Fail & Fail & Fail & Fail & Fail & Pass & Pass & Pass & Pass & Pass & Pass & Pass & Pass & Fail & Fail & Fail & Fail & Fail & Fail & Fail & Fail \\
\hline
\end{tabular}

*Image noise is mean from 10 scans

$\dagger 15 \%$ output change corresponds to $3.9 \%$ change in image noise, $95 \%$ CI for image noise $=[5.1,5.7]$. CI was calculated by using the CI for baseline noise and applying scaling factor to calculate $\mathrm{CI}$ at $+/ 15 \%$ output deviation from baseline. The lower limit of the CI for $15 \%$ output and the upper limit of the CI for $+15 \%$ output were used in the final CI.

FIG. 2: Results of QC experiment demonstrate that it is possible to detect changes in post replacement tube output using historical QC data. Values in the top row highlighted green indicate output changes that are within $+/-15 \%$ manufacturer limits, values highlighted in red fall outside limits. "Pass" or "Fail" values in bottom row indicate results of comparison of image noise between "post change" images, simulated by changing $\mathrm{mA}$, and "pre change" historical QC data. One output value that was within specified limits (-15\%) was indicated as failing by our analysis. It was noted that changes in image noise were larger than expected as output decreased, while changes were at the expected level as output was increased. One possible explanation for this result is increasing contribution of electronic noise as output was reduced. 